Pathologe 2021 · 42:1

https://doi.org/10.1007/s00292-020-00890-w

Angenommen: 14. Dezember 2020

(c) Springer Medizin Verlag GmbH, ein Teil von Springer Nature 2021

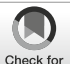

Check for
updates

Liebe Leserinnen und Leser,

liebe Kolleginnen und Kollegen,

in meiner alltäglichen diagnostischen Tätigkeit hat die Zeitschrift Der Pathologe immer einen festen Platz, und zwar in Form von wachsenden Stapeln von Themenheften direkt neben dem Mikroskop. Meiner Erfahrung nach sind in zahlreichen Fällen die gesuchten Informationen weitaus schneller und einfacher durch einen gezielten Griff zu einem Übersichtsartikel in Der Pathologe verfügbar als durch Nachschlagen in dicken Lehrbüchern oder (sogar!) als durch die digitale Internetsuche. Die gedankliche Assoziation beim Mikroskopieren: „Da gab es doch mal einen Review von ... im Pathologen" hat zumindest bei mir in vielen Fällen zur zielgerichteten Klärung, Absicherung und Beschleunigung von Diagnosen geführt. Daher sehe ich eine primäre Aufgabe des Pathologen darin, eine für die alltägliche Praxis relevante "desk top reference“ zu sein, verbunden mit pathologiebezogenen Hintergrundinformationen zu wichtigen und aktuellen Themen.

Dass Der Pathologe einen solchen Stellenwert erreichen konnte, ist vor allem der wesentliche Verdienst einer langjährigen Reihe von engagierten Schriftleitern und Editorial Boards. In den letzten 10 Jahren wurde die Zeitschrift wesentlich von Kurt W. Schmid geprägt, der durch die Einführung einiger neuer Rubriken das Erscheinungsbild attraktiver und die Ausrichtung praxisrelevanter gemacht hat, wie z. B. die Rubriken „Pitfalls", „CME-zertifizierte Fortbildungen“ oder die „Standortbestimmung Pathologie". Für das Selbstverständnis der Pathologie hat die von ihm ins Leben gerufene Beitragsserie „Geschichte der Patho-

Wilfried Roth

Institut für Pathologie, Universitätsmedizin Mainz, Mainz, Deutschland

\title{
Zur Übernahme der Schriftleitung
}

logie" eine ganz besondere Bedeutung. An dieser Stelle möchte ich Kurt Schmid für seinen unermüdlichen Einsatz und seine wertvollen Impulse ganz herzlich danken! Die Erfolgsgeschichte von Der Pathologe in der letzten Dekade verdankt sich der konstruktiven Zusammenarbeit von Kurt Schmid und seinem langjährigen Editor Hideo Baba mit dem gesamten Editorial Board der Zeitschrift und vor allem auch mit den engagierten Mitarbeiterinnen des Springer-Verlags.

Nach mittlerweile über 40-jährigem Erscheinen von Der Pathologe bin ich mir der zukünftigen Herausforderungen und der Notwendigkeit des Wandels unter sich dauernd ändernden Bedingungen sehr bewusst. Das Thema „Digitalisierung" wird auch in diesem Bereich zentral sein. Und die - mittlerweile eher ungewöhnliche - Ausrichtung von Der Pathologe als deutschsprachige edukative Zeitschrift in einem immer mehr globalisierten, kompetitiven Publishingmarkt wird besondere Anforderungen mit sich bringen. Diese Herausforderungen werden wir am besten meistern können, wenn es zu einem lebendigen, vielfältigen Austausch zwischen Herausgebern, Verlag, kooperierenden Gesellschaften und der gesamten Leserschaft kommt. In diesem Sinne bitte ich Sie alle ausdrücklich, mit Ideen, Wünschen und Kritik jederzeit auf mich zuzukommen!

\section{Mit besten Grüßen}

Ihr

Wilfried Roth

\section{Korrespondenzadresse}

Prof. Dr. Wilfried Roth

Institut für Pathologie, Universitätsmedizin Mainz

Langenbeckstr. 1, 55131 Mainz, Deutschland wilfried.roth@unimedizin-mainz.de

Interessenkonflikt. W. Roth gibt an, dass kein Interessenkonflikt besteht.

\begin{tabular}{l}
\hline Der neue federführende \\
Schriftleiter \\
\hline \\
Prof. Dr. Wilfried Roth wurde 1970 in \\
Tettnang geboren. Nach der Schulausbildung \\
und einem kurzen Ausflug in die \\
Geisteswissenschaften studierte er Medizin \\
in Tübingen. Es folgten eineinhalb Jahre \\
als Assistenzarzt in der Neurologie in \\
Tübingen und anschließend ein mehrjähriger \\
wissenschaftlicher Postdoc-Aufenthalt in \\
San Diego, USA. Nach der Rückkehr nach \\
Deutschland und einer kurzen Zeit in der \\
Neuropathologie in Bonn absolvierte er \\
seine Facharztausbildung für Pathologie \\
in Heidelberg, wo er sich 2008 habilitierte. \\
Parallel zur Facharztausbildung leitete er eine \\
von der Deutschen Krebshilfe geförderte \\
Max-Eder-Nachwuchsgruppe am DKFZ in \\
Heidelberg. Im Jahr 2009 Verleihung des \\
Rudolf-Virchow-Preises der Deutschen \\
Gesellschaft für Pathologie. Ab 2011 \\
Professur für Molekulare Tumorpathologie \\
am Universitätsklinikum Heidelberg und \\
DKFZ sowie Leitung der gleichnamigen \\
Klinischen Kooperationseinheit am DKFZ \\
und am Pathologischen Institut. Ab 2015 \\
Direktor des Institutes für Pathologie an der \\
Universitätsmedizin Mainz. \\
\hline
\end{tabular}

